\title{
Associations between Individual-level and Area-level social disadvantage and oral health behaviours in Australian adolescents
}

\author{
Nadia Kaunien, ${ }^{1}$ Ankur Singh, ${ }^{1}$ Tania King ${ }^{1}$
}

1. Centre for Health Equity, Melbourne School of Population and Global Health, University of Melbourne, Melbourne, Australia

\section{Corresponding author: Nadia Kaunein}

Level 4, 207 Bouverie St. Melbourne School of Population and Global Health.

The University of Melbourne. Victoria 3010. Australia.

Ph: +61415500464. email: nadia.kaunein@unimelb.edu.au or drkaunein@gmail.com

\section{Acknowledgements}

This article uses data from the Longitudinal Study of Australian Children (LSAC), a survey conducted in partnership between the Department of Social Services, the Australian Institute of Family Studies and the Australian Bureau of Statistics. We thank all the young people and their families that participated in LSAC. Everyone who contributed significantly to the work have been mentioned.

\section{Funding}

This research did not receive any specific grant from funding agencies in the public, commercial, or not-for-profit sectors.

Tania King is supported by an Australian Research Council Discovery Early Career Researcher Award(DE200100607).

\section{Conflict of interest: None}

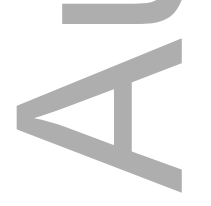

This is the author manuscript accepted for publication and has undergone full peer review but has not been through the copyediting, typesetting, pagination and proofreading process, which may lead to differences between this version and the Version of Record. Please cite this article as doi: $10.1111 /$ ADJ.12792

This article is protected by copyright. All rights reserved 


\section{Author contributions}

The first draft of the manuscript was written by the first author, Nadia Kaunein. No honorarium, grant, or other form of payment was given to anyone to produce the manuscript.

Nadia Kaunein: Conceptualisation, Formal analysis, Investigation, Methodology, Validation, Writing - Original draft, review \& editing.

Tania King: Conceptualisation, Investigation, Methodology, Supervision, Validation, Writing- review \& editing.

Ankur Singh: Conceptualisation, Investigation, Methodology, Supervision, Validation, Writing- review \& editing.

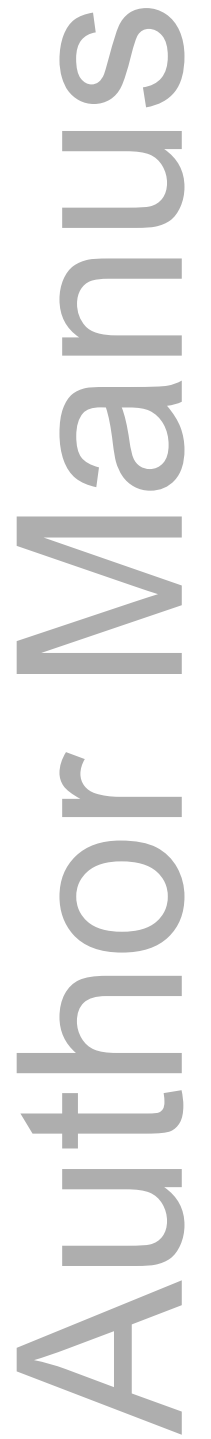




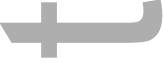 \\ Article type : Scientific Article

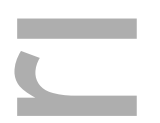 \\ Associations between Individual-level and Area-level social disadvantage and oral health behaviours in Australian adolescents
}

\begin{abstract}
Background: Oral health behaviours are significant determinants of oral health. There is evidence that socio-economic position influences oral health behaviours, but little is known about this association during adolescence. This study aims to investigate the association between social disadvantage (individual- and area-level) and oral health behaviours among Australian adolescents.
\end{abstract}

Methods: This study utilised data from the Longitudinal Study of Australian Children (LSAC). The sample consisted of 2877 adolescents. Exposure measures were area-level social disadvantage (Socioeconomic Indexes for Areas (SEIFA)), and parent-reported household income from Wave 5. Outcomes, measured in Wave 6, wereadolescent-reported oral health behaviours: frequency of brushing, consumption of sugar-sweetened beverages (SSB) and dental visits. Associations between quintiles of each exposure and each oral health behaviour were tested by fitting multivariable logistic regression models.

Results: Household income and area-level disadvantage were associated with dental visits and brushing frequency. Associations between social disadvantage and consumption of SSBs was less apparent, with only the leastdisadvantaged adolescentshaving lower odds of consumption of SSBs compared to the mostdisadvantaged group. 
Conclusion: Broad population-based strategies must be adopted to reduce intake of SSBs, however targeted strategies are needed among more disadvantaged populations to address infrequent tooth brushing and irregular dental visits among adolescents.

Keywords: Adolescence, Australia, oral health behaviours, SEIFA, social disadvantage.

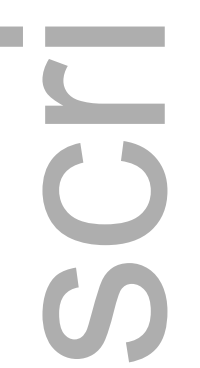

\section{BACKGROUND}

Globally, over 3.9 billion people experience oral diseases, with dental caries being the most common condition according to the Global Burden of Disease Study 2015(1). Over $\$ 8$ billion AUD (Australian Dollars) is spent yearly on dental services, with dental caries being the second most expensive diet-related disease(1). Oral diseases significantly contribute to the burden of disease worldwide and in Australia. In addition to causing pain, discomfort, financial burden and reduced quality of life, dental caries and periodontal diseases together account for $90 \%$ of tooth loss(1). The impact of oral diseases is not just limited to the individual, but also extends to society at large through high financial costs and a burdened health system(2). The burden of oral diseases is also higher among socially disadvantaged individuals than among their more advantaged counterparts(3).

Most prevalent oral health conditions, including dental caries and periodontal diseases, are largely preventable as oral health behaviours play an essential role in their aetiology(4). There is strong evidence that sugar is an important cause of dental caries. The World Health Organisation (WHO), recommends lowering free sugar consumption to 5\% of total energy intake to reduce the detrimental impact of sugar on dental health (5). Consumption of free sugars, particularly in the form of sugar-sweetened beverages, has been consistently linked with rising levels of dental caries(6). Sugar-sweetened beverages (SSB) by definition include drinks with added sugar such as, fruit drinks, soft drink and energy drinks(6). Sugars, especially in the form of SSBs can be easily consumed in large quantities in the current food environment. For instance, an average cola drink can contains 40 grams of free sugars 
(approximately10 teaspoons of sugar)(7). Despite recommendations by health organisations to reduce consumption of SSBs, sales of soft drinks and other SSB's are increasing worldwide, further contributing to the burden of disease (8) .

Preventive and regular dental visits are also known to benefit dental health by reducing the progression of oral diseases, and are therefore key to the prevention of dental caries(9). Similarly, oral hygiene practices, primarily tooth brushing twice or more during the day with fluoridated toothpaste, are known to reduce the occurrence of dental caries due to local delivery of fluoride. Additionally, by physically removing plaque and calculus, toothbrushing also prevents periodontal disease(4). Other risk factors for oral diseases include tobacco smoking and alcohol use(10).

Adolescence is a key stage of life, and is particularly important in terms of the establishment and maintenance of health behaviours(11). Investigating health behaviours in this age group is crucial, as many patterns for future health-related behaviours and outcomes have their roots in adolescence(12). Additionally, in relation to oral health, adolescence is a vital period as permanent dentition is complete, and this is when parental monitoring of oral health behaviour typically ceases(13). Further, evidence shows that poor dental health in adolescence is a risk factor for poor dental health in adulthood(14). The period of adolescence, therefore, poses an optimal point of intervention to prevent the effects of poor oral health behaviours being carried into adulthood.

Although there have been studies examining theassociations between social disadvantage and oral health behaviours among adolescentsin countries such as New Zealand(15, 16), Brazil(17) and the United states of America(18), little examination has been done in Australia. Additionally, this is the first study that we are aware of that has analysed both individual-level and area-level social disadvantage in relation to oral health behaviours among adolescents in Australia.Given the importance of the adolescent period in establishing good health trajectories, any observed associations between social disadvantage and oral health could be exacerbated across the life-course. Additionally, examining and identifying associations between social disadvantage and oral health behaviours are needed to tailor preventive strategies. Using data from a representative sample of Australian adolescents aged 14-15 years, this study aimed to test whether individual- and area-level social disadvantage is associated with three oral health behaviours (SSB consumption, frequent tooth brushing and regular dental attendance). 


\section{METHODS}

\section{Study population}

This study utilised data from the 'Longitudinal Study of Australian Children'(LSAC)(19), which has a multiple cohort cross-sequential study design. The study received ethics approval from the Australian Institute of Family Studies Ethics Committee and conforms to the principles embodied in the Declaration of Helsinki.Data from Waves 4, 5 and 6, used in this research were collected between 2010 and 2014, by face-to-face interviews and Computer Assisted Self Interviews at the participant's home every two years. Participants in this study include adolescents aged 14 to 15 years. The eligible sample of adolescents in Cohort $\mathrm{K}$ were 3240. Those with missing data on covariates, and exposure and outcome variables were excluded, resulting in an analytic sample of 2877 adolescents. To respect the theorised temporal relationship between the variables, covariates were obtained from Wave 4 (Year: 2010), exposures from Wave 5 (Year: 2012) and outcomes from Wave 6 (Year: 2014).

\section{Exposure}

Socio-economic position (SEP) at an individual level was measured using parent-reported household income measured in Australian dollars (AUD). This continuous variable was mean centred and categorised into quintiles. The second exposure, area-level SEP, was measured using the Socio-Economic Indexes for Areas (SEIFA). SEIFA is developed by the Australian Bureau of Statistics (ABS) and measures socio-economic advantage and disadvantage at an area level (20). This study used SEIFA - IRSD from 2011 obtained from the ABS. This continuous variable was also converted into quintiles.

\section{Outcomes}

Three oral health behaviours were included in the study: dental visits, consumption of SSBs and tooth brushing. Regarding dental visits, participants were asked "Have you visited the dentist in the last 12 months?' and eligible responses were 'Yes' or 'No'(21). For SSBs, participants were asked, "Thinking about yesterday, how often did you have... soft drink or cordial, not diet or sugar free soft drink or cordial?". Available responses were: not at all, once, twice or more. These responses were dichotomised for analysis: 'once' and 'twice or more' were combined as one category with 'not at all' as the reference category(22). Regarding tooth brushing, participants were asked, "how often did you clean your teeth?". Eligible responses were: not at all, once, twice and more than twice. Responses were 
dichotomised with 'not at all' and 'once' combined to represent infrequent tooth brushing, while 'twice' and 'more than twice' were combined for the reference category(22).

\section{Covariates}

Covariates included in the models as confounders were gender (male, female), parental education ('at least one parent completed year 12' and 'no parent completed year 12') geographic remoteness (non-remote and remote areas: the non-remote category comprised of major cities, inner regional areas, and outer regional areas while the remote category consisted of remote areas and very remote areas), household composition (single parent/caregiver household, household with two parents) and Indigenous status (Indigenous Australian identity, non-Indigenous Australian identity). All confounding variables were dichotomised.

\section{Statistical Analysis}

Multivariable logistic regression models were fitted to test the associations between both exposures and each oral health behaviour after adjusting for confounding variables. Separate models were fitted for household income and each oral health behaviour (Model 1), and for area-level SEP and each oral health behaviour (Model 2). Model 3 mutually adjusted for both household income and area-level SEP in the same model. All models were adjusted for gender, parental education, geographic remoteness, household composition, and Indigenous status. To account for the complex survey design, survey commands (prefix: svy) were used. All statistical analyses were performed in Stata version 15. (StataCorp. 2017. Stata Statistical Software: Release 15. College Station, TX: StataCorp LLC).

\section{RESULTS}

The sample had equal representation of males and females with most adolescents identifying as non-Indigenous $(97.3 \%)$, belonging to households with two parents (80\%) and living in non-remote areas (98.8\%). In relation to parental education, two-thirds of the sample had at least one parent who had completed year 12 education (63.3\%). Overall, 35.8\% of adolescents had not visited a dentist in the last 12 months, $33.4 \%$ of the sample did not brush at least two times a day and almost $71.5 \%$ had consumed sugar-sweetened beverages at least once in the last 24 hours (Table 1).

In analytic models, a clear gradient was observed between both exposures (household income and SEIFA) and dental visits. The odds of visiting the dentist were $77 \%$ higher in adolescents from highest income quintiles (quintiles 4 and 5) when compared to those from lowest 
income quintile (OR 1.76 95\%CI 1.29-2.41; OR 1.77, 95\%CI 1.30-2.43 respectively). Similar associations were observed for area-level SEP, with those in the least disadvantaged quintiles (quintiles 4 and 5) reporting greater odds of visiting the dentist (OR 1.48 95\%CI 1.09-2.01; OR 1.84 95\%CI 1.38-2.47). These associations persisted with the inclusion of both exposures in Model 3 (Table 2).

Table 3 shows associations between the exposure variables and frequency of tooth brushing. Those in income quintile 4 and 5 (highest household income) had greater odds of brushing at least twice a day, compared to those in the lowest quintile (OR 1.39 95\%CI 1.02-1.91, OR 1.35 95\% CI 1.00-1.82 respectively). These associations did not persist when models also adjusted for area-level SEP (model 3). In relation to area-level SEP, those in quintiles 2-5 had approximately $50 \%$ increased odds of brushing at least two times a day compared to those in the most disadvantaged quintiles (OR 1.50 95\%CI 1.13-2.01; OR 1.57 95\%CI 1.15-2.13; OR 1.50 95\%CI 1.14-1.97 quintile 2, quintile 4, quintile 5 respectively). These associations remained after adjusting for household income in model 3.

Associations betweensocial disadvantage (household income or SEIFA score) and the consumption of sugar-sweetened beverages in adolescents (Table 4) were only observed for the least disadvantaged quintile (quintile 5) $(\mathrm{OR}=0.7195 \% \mathrm{CI} 0.53-0.97$ for income in model 3) and $(\mathrm{OR}=0.7095 \% \mathrm{CI} 0.51-0.96$ for area-based SEP in model 3). This was consistent in all three models, and no gradient was observed.

\section{DISCUSSION}

This study presents evidence that individual-level and area-level socioeconomic position is associated with tooth brushing and regular dental attendance among Australian adolescents. Individual-level socioeconomic position and area-level socioeconomic position were associated with SSB consumptiononly in the least disadvantaged group of adolescents.

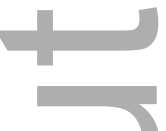

The findings that individuals from higher socio-economic position are more likely to regularly visit the dentist compared to their disadvantaged counterparts are in line with previous research among adults (23). The most common deterrent to regular dental visits is the high cost of dental services(23). Other factors include the disproportionate number of dental practices in least disadvantaged areas when compared to most disadvantaged areas, 
lack of public transport, and lack of knowledge regarding the importance of preventative dental treatment(23).

No prominent association or social gradient was observed between socio-economic position, measured at either the individual or area-level, and the consumption of sugar-sweetened beverages.However, adolescents from higher socio-economic position had lower odds of consumption of SSB.This could partly be explained because adolescents aged 14 to 15 years consume the highest levels of SSBs of any age group in Australia(24) and the low cost of soft drinks, and cordial makes consumption affordable and accessible. Indeed, SSBs are cheaper than bottled water in many countries including Australia(25) and the perception that SSBs are good value for money further drives consumption(26). SSBs are also often easily available at school vending machines and school canteens, further increasing accessibility(27). Furthermore, it is widely believed that fruit juices and sports beverages (neither of which are captured here) are healthy, this also likely driving consumption of sugar in beverages(4).

There have been conflicting reports in relation to individual socio-economic position and SSBs, with some studies demonstrating an association between lower socio-economic position and an increased intake of sugar-sweetened beverages $(28,29)$. The findings related to area-level disadvantage are also in contrast to previous research which suggests that individuals residing in more disadvantaged areas consume more sugar-sweetened beverages(30). A better understanding how area-level social disadvantage impacts individual SSB consumption is needed. We argue however, that it is likely that the pervasive and high supply of SSBs makes the whole population of adolescents vulnerable regardless of their level of social disadvantage - this potentially underpinning the fact that the only association between SEP and SSB consumption observed was for the most advantaged (least disadvantaged) quintile.

Two key features of the afore-mentioned oral health behaviours underscore the imperatives to understand and address them. First, they are also common risks to a range of general health conditions. For example, high sugar intake is associated with obesity and diabetes(31). Therefore, addressing oral health behaviours have twofold benefits of reducing oral diseases and preventing life threatening general health conditions. Second, more socially disadvantaged individuals are more vulnerable to health compromising behaviours than their advantaged counterparts(32). Improving oral health behaviours may represent one opportunity to partly reduce unfair social inequalities in oral health outcomes. 
The results showing a weak association between tooth brushing behaviour in adolescents and household income is broadly consistent with previous research showing that individuals from low-income households are more likely to report infrequent tooth brushing behaviour(33). However, many prior studies have not adjusted for area-level disadvantage. Hence the findings from this paper build on this existing research by demonstrating that the association observed between household income and brushing frequency may be largely driven by arealevel disadvantage.

Possible explanations for the association between area-level disadvantage and tooth brushing include the role of social processes such as social capital that may influence social norms related to tooth brushing or generally healthy behaviours in areas(34). Social capital is defined as "features of social organization such as trust, norms, and networks that can improve the efficiency of society by facilitating coordinated actions"(35, p 167). Areas with high social capital have a more cohesive neighbourhood with more trust, mutual concern for others and health-promoting behaviour(36). Further, these neighbourhoods also commonly have a shared consensus regarding what constitutes 'appropriate' practices and behaviours, which in turn influences and encourages good health behaviours. On the other hand, areas with low levels of social capital tolerate health-compromising behaviours due to a less binding moral order(37). Further work will examine the role of social capital in the association between area-level social disadvantage and tooth brushing.

We note some important strengths and limitations of this work. This is the first study that we are aware of that has analysed both individual-level and area-level social disadvantage in relation to oral health behaviours among adolescents in Australia. In addition, the longitudinal design of the study is an advantage, enabling us to define the theorised temporal ordering of the exposure and outcome. Further, a large population representative sample was used, with good retention of participants. In terms of limitations, the lack of information regarding the reason for dental visits and quantity of sugar-sweetened beverages consumed was a limitation.Dental visits could be related to dental trauma, be treatment focused or be preventative in nature; this information could provide more clarity regarding good oral health behaviour. Similarly, information on the quantity of sugar-sweetened beverages consumed would be of benefit, as there is some evidence of a dose-response relationship between the consumption of sugar-sweetened beverages and dental caries(38). A more detailed examination of the consumption patterns of SSB in adolescents is needed, with particular attention to the quantity as well as the frequency of consumption. 
Poor oral health behaviours observed in the most disadvantaged individuals (at both the individual and area-level) supports the argument that oral health is socially determined. The challenge is, however, to identify an appropriate solution for these social inequalities.

Interventions to reduce oral health inequalities may be best directed at all levels, including at individual behaviours, as well as upstream interventions at a community or population level. For example, increasing the federal budget for public dental care and universal dental health coverage for the population may reduce inequalities. In addition, increasing the availability of, and access to, dental clinics in the most disadvantaged neighbourhoods to improve access to regular and preventative dental care is essential, andmay be important in addressing the contextual factorsrelated to dental health inequalities. The introduction of school-based tooth brushing programs may also be beneficial, as this would target all students, irrespective of their social background. Additionally, the free distribution of fluoridated toothpastes in schools and other education or sports centres where adolescents frequently visit may also be effective. Further, policies to remove SSBs from vendors in close proximity to schools should be implemented to reduce easy access to SSBs.

The World Health Organisation recommends a sugar tax as an effective intervention to reduce consumption of sugars (39), just as taxing tobacco has helped to significantly reduce tobacco use (40). Taxation on SSBs in the United States of America has shown promising results, delivering a US\$ 17 billion in healthcare cost savings(41). In Mexico, implementation of a sugar tax led to low-income consumers reducing purchases of SSBs by $11.7 \%$ (42).

Moreover, revenue generated by these taxes could be used to improve health care systems (43).

Effective dental policies targeting adolescents at both the individual and community level are vital- not simply to create healthy oral behaviours that can be carried into adulthood, but also to reduce health inequalities early in life.

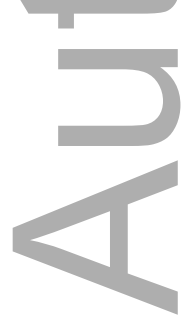

\section{REFERENCES}


[1] Kassebaum NJ, Smith AGC, Bernabe E, et al. Global, Regional, and National Prevalence, Incidence, and Disability-Adjusted Life Years for Oral Conditions for 195 Countries, 1990-2015: A Systematic Analysis for the Global Burden of Diseases, Injuries, and Risk Factors. J Dent Res 2017;96:380-387.

[2] Rogers J. Evidence-based oral health promotion resource. In: Services DoHH, ed., 2011.

[3] Watt RG, Heilmann A, Listl S, et al. London Charter on Oral Health Inequalities. J Dent Res 2016;95:245-247.

[4] Ranjit N, Evans MH, Byrd-Williams C, et al. Dietary and activity correlates of sugar-sweetened beverage consumption among adolescents. Pediatrics 2010;126:e754-761.

[5] WHO. Guideline on sugars intake for adult and children. 2015.

[6] Bleich SN, Vercammen KA. The negative impact of sugar-sweetened beverages on children's health: an update of the literature. BMC Obesity 2018;5:6.

[7] Worldwide trends in body-mass index, underweight, overweight, and obesity from 1975 to 2016: a pooled analysis of 2416 population-based measurement studies in 128.9 million children, adolescents, and adults. Lancet 2017;390:2627-2642.

[8] Kleiman S, Ng SW, Popkin B. Drinking to our health: can beverage companies cut calories while maintaining profits? Obes Rev 2012;13:258-274.

[9] Bhaskar V, McGraw KA, Divaris K. The importance of preventive dental visits from a young age: systematic review and current perspectives. Clin Cosmet Investig Dent 2014;6:21-27.

[10] Rooban T, Vidya K, Joshua E, et al. Tooth decay in alcohol and tobacco abusers. J Oral Maxillofac Pathol 2011;15:14-21.

[11] Viner R, Macfarlane A. Health promotion. BMJ 2005;330:527-529.

[12] Achieving universal health coverage for the world's 1.2 billion adolescents. Available at: https://www.who.int/maternal child adolescent/adolescence/universal-health-coverage/en/. [13] Jung S-H, Kim M-H, Ryu J-I. Inequalities in oral health among adolescents in Gangneung, South Korea. BMC Oral Health 2018;18:68.

[14] Australia's Adult Oral Health Tracker: Australian Dental Association; 2018.

[15] Broadbent JM, Zeng J, Foster Page LA, et al. Oral Health-related Beliefs, Behaviors, and Outcomes through the Life Course. J Dent Res 2016;95:808-813.

[16] Broadbent JM, Thomson WM, Poulton R. Oral health beliefs in adolescence and oral health in young adulthood. J Dent Res 2006;85:339-343.

[17] Freire MdCM, Jordão LMR, Malta DC, et al. Socioeconomic inequalities and changes in oral health behaviors among Brazilian adolescents from 2009 to 2012. Rev Saude Publica 2015;49:50-50.

This article is protected by copyright. All rights reserved 
[18] Polk DE, Weyant RJ, Manz MC. Socioeconomic factors in adolescents' oral health: are they mediated by oral hygiene behaviors or preventive interventions? Community Dent Oral Epidemiol 2010;38:1-9.

[19] Department of Social Services, Australian Institute of Family Studies, Australian Bureau of Statistics. Growing Up in Australia: Longitudinal Study of Australian Children (LSAC) Release 6 (Waves 1-6). ADA Dataverse, 2020.

[20] Australian Bureau of Statistics. Socio-Economic Indexes for Areas (SEIFA) - Technical Paper. Canberra: ABS, 2016.

[21] Peltzer K, Pengpid S. Oral health behaviour and social and health factors in university students from 26 low, middle and high income countries. Int J Environ Res Public Health 2014;11:1224712260.

[22] Singh A, Rouxel P, Watt RG, et al. Social inequalities in clustering of oral health related behaviors in a national sample of British adults. Prev Med 2013;57:102-106.

[23] Sanders AE. Social determinants of oral health : conditions linked to socioeconomic inequalities in oral health in the Australian

population. Canberra; 2007.

[24] ABS. Australian Health Survey: Nutrition First Results - Foods and Nutrients, 2011-12. Canberra: Australian Bureau of Statistics, 2014.

[25] Firger J. Sugar-Sweetened Beverages are Now Cheaper than Bottled Water in Many Countries. Newsweek Magazine, 2017.

[26] Scully M, Morley B, Niven P, et al. Factors associated with high consumption of soft drinks among Australian secondary-school students. Public Health Nutr 2017;20:2340-2348.

[27] Park S, Blanck HM, Sherry B, et al. Factors associated with sugar-sweetened beverage intake among United States high school students. J Nutr 2012;142:306-312.

[28] Backholer K, Sarink D, Beauchamp A, et al. The impact of a tax on sugar-sweetened beverages according to socio-economic position: a systematic review of the evidence. Public Health Nutr 2016;19:3070-3084.

[29] Bolt-Evensen K, Vik FN, Stea TH, et al. Consumption of sugar-sweetened beverages and artificially sweetened beverages from childhood to adulthood in relation to socioeconomic status 15 years follow-up in Norway. Int J Behav Nutr Phys Act 2018;15:8-8.

[30] Thurber K, Bagheri N, Banwell C. Social determinants of sugar-sweetened beverage consumption in the Longitudinal Study of Indigenous Children. In: Australian Institute of Family Studies, ed.: Family Matters, 2014:51-61. 
[31] Kassebaum NJ, Smith AGC, Bernabe E, et al. Global, Regional, and National Prevalence, Incidence, and Disability-Adjusted Life Years for Oral Conditions for 195 Countries, 1990-2015: A Systematic Analysis for the Global Burden of Diseases, Injuries, and Risk Factors. Journal of dental research 2017;96:380-387.

[32] Bor W, Najman JM, Andersen M, et al. Socioeconomic disadvantage and child morbidity: an Australian longitudinal study. Soc Sci Med 1993;36:1053-1061.

[33] Freddo Silvia Letícia, Aerts Denise Rangel Ganzo de Castro, Abegg Claídes, Davoglio Rosane, Vieira Patrícia Conzatti, Monteiro Lisiane. Oral hygiene habits and use of dental services among schoolchildren in a city in the South of Brazil. Cad. Saúde Pública [Internet]. 2008 Sep [cited 2020 Aug 04]; 24 (9): 1991-2000. Available from:

http://www.scielo.br/scielo.php?script=sci_arttext\&pid=S0102311X2008000900005\&lng=en. https://doi.org/10.1590/S0102-311X2008000900005..

[34] Pattussi MP, Marcenes W, Croucher R, et al. Social deprivation, income inequality, social cohesion and dental caries in Brazilian school children. Soc Sci Med 2001;53:915-925.

[35] Putnam RD, Leonardi R, Nanetti R. Making Democracy Work: Civic Traditions in Modern Italy. Princeton: Princeton University Press, 1993.

[36] Putnam RD. Social Capital: Measurement and Consequences. Isuma: Canadian Journal of Policy Research 2001;2.

[37] Kawachi I, Kennedy BP, Glass R. Social capital and self-rated health: a contextual analysis. Am J Public Health 1999;89:1187-1193.

[38] Nguyen T. Is the current model of public dental care promoting the oral health of young children in Australia? Deeble Institute Issues Brief Paper No. 20. 2017.

https://ahha.asn.au/sites/default/files/docs/policy-

issue/deeble institute paper 20 oral health.pdf. Accessed 05 Aug,2020.

[39] Bernabe E, Vehkalahti MM, Sheiham A, et al. The Shape of the Dose-Response Relationship between Sugars and Caries in Adults. J Dent Res 2016;95:167-172.

[40] 'Best buys' and other recommended interventions for the prevention and control of noncommunicable diseases. Geneva: World Health Organisation; 2017.

[41] Sharbaugh MS, Althouse AD, Thoma FW, et al. Impact of cigarette taxes on smoking prevalence from 2001-2015: A report using the Behavioral and Risk Factor Surveillance Survey (BRFSS). PLOS ONE 2018;13:e0204416.

[42] Wang YC, Coxson P, Shen YM, et al. A penny-per-ounce tax on sugar-sweetened beverages would cut health and cost burdens of diabetes. Health Aff (Millwood) 2012;31:199-207.

This article is protected by copyright. All rights reserved 
[43] Colchero MA, Rivera-Dommarco J, Popkin BM, et al. In Mexico, Evidence Of Sustained Consumer Response Two Years After Implementing A Sugar-Sweetened Beverage Tax. Health Aff (Millwood) 2017;36:564-571.

[44] Taxes on sugary drinks: Why do it?: World Health Organisation; 2017.

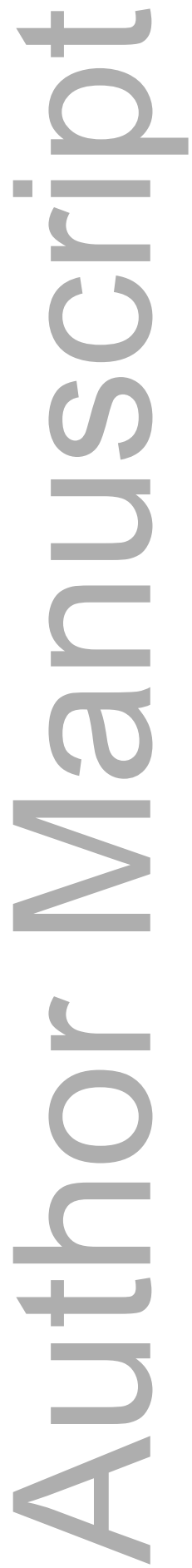


Table 1: Descriptive characteristics of the sample $(n=2877)$

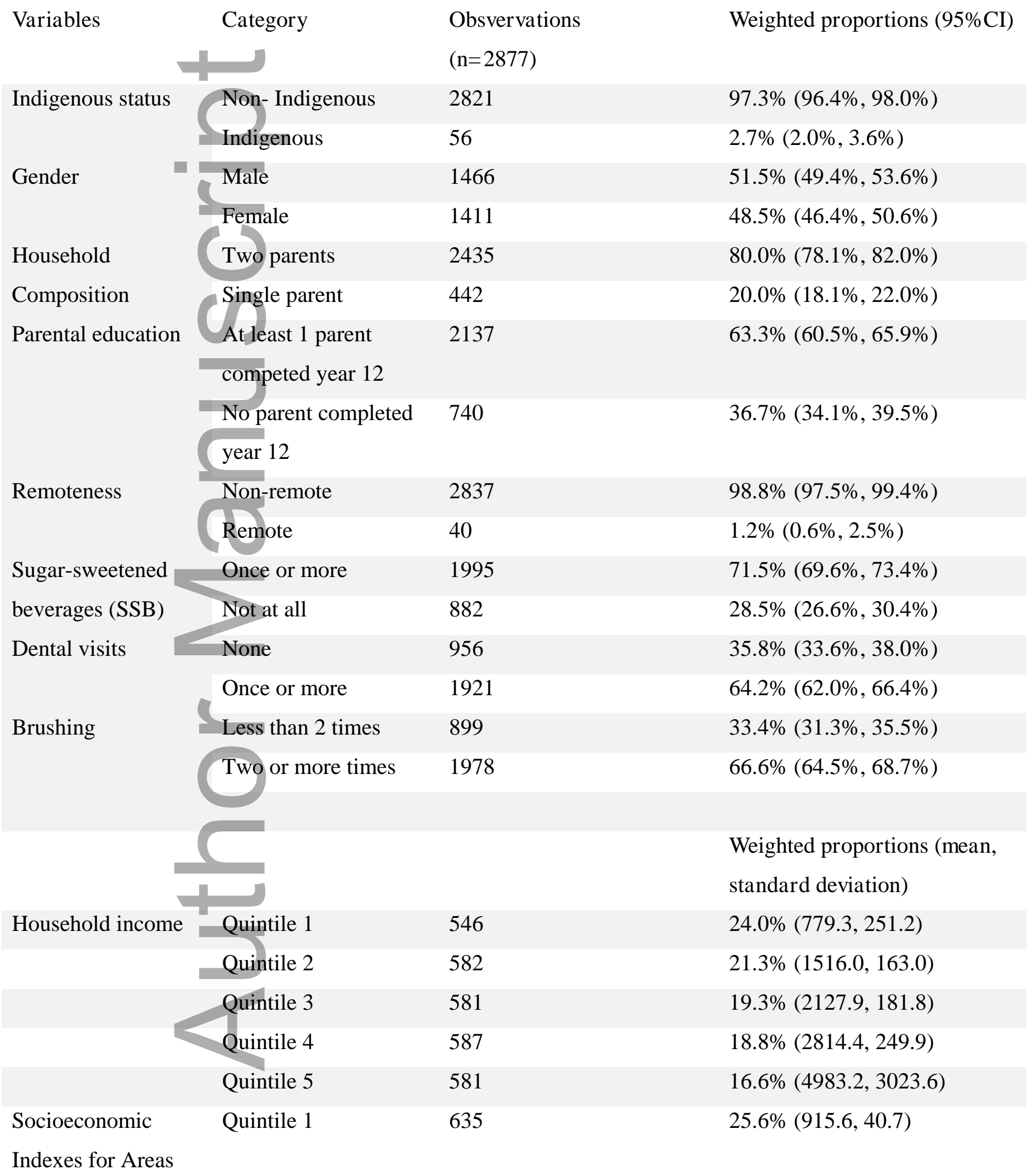


(SEIFA) Score

Quintile 2

Quintile 3

Observations $(\mathrm{n}=2877)$

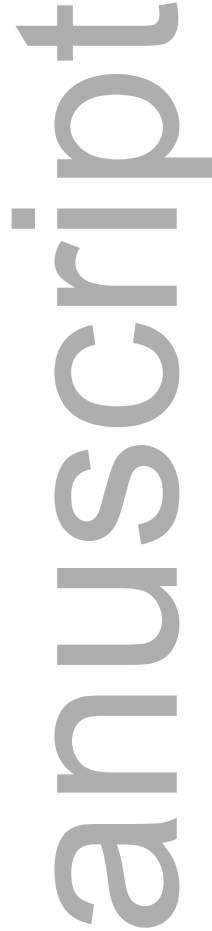

Quintile 4

Quintile 5

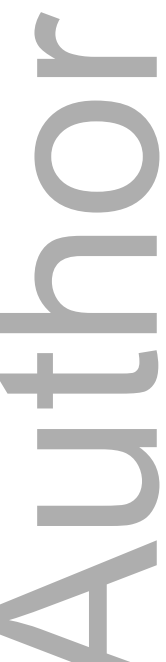

523

639

Model 1*

$19.1 \%(985.2,10.4)$

$21.2 \%(1024.2,11.3)$

Model 3***
533

547
$17.0 \%(1061.2,8.5)$

$17.0 \%(1097.0,15.1)$

Table 2: Multivariable logistic regression models for the associations between household income, Socioeconomic Indexes for Areas (SEIFA) score and dental visits.

This article is protected by copyright. All rights reserved 


\section{OR $(95 \% \mathrm{CI}) \quad$ OR $(95 \% \mathrm{CI}) \quad$ OR $(95 \% \mathrm{CI})$}

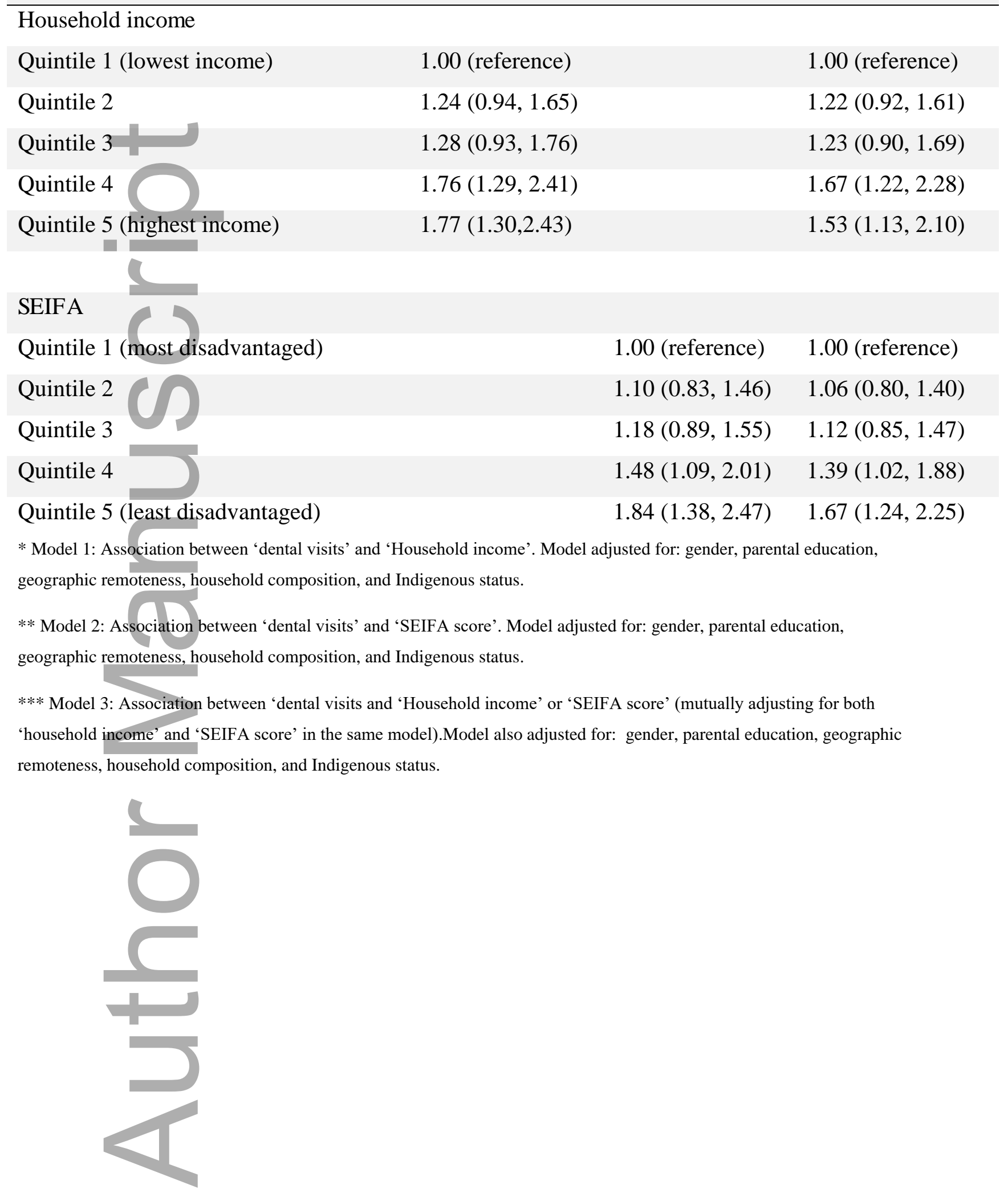


Table 3: Multivariable logistic regression models for the associations between household income, Socioeconomic Indexes for Areas (SEIF A) score and tooth brushing habits.

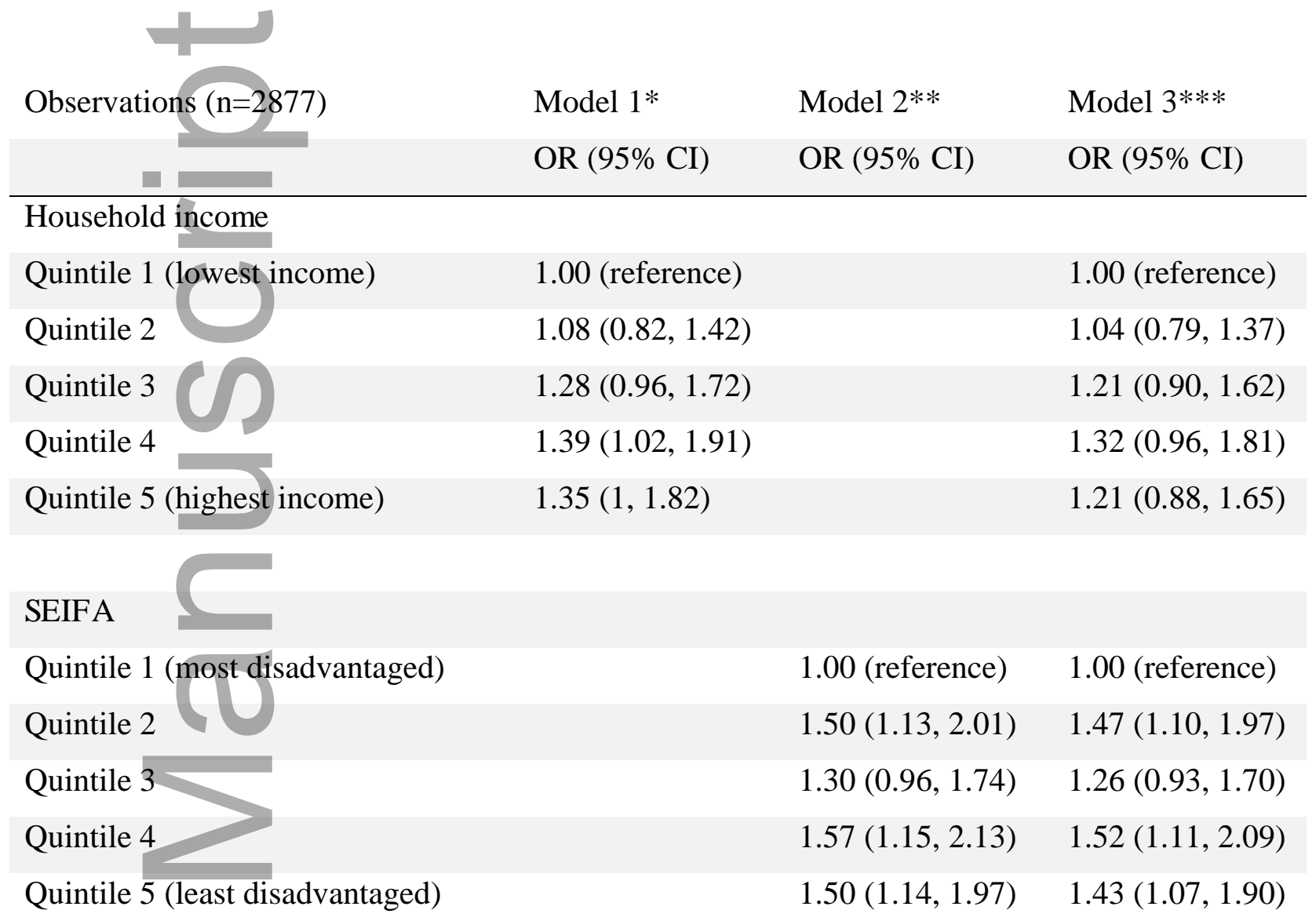

* Model 1: Association between 'tooth brushing habits' and 'Household income'. Model adjusted for: gender, parental education, geographic remoteness, household composition, and Indigenous status.

** Model 2: Association between 'tooth brushing habits' and 'SEIFA score'. Model adjusted for: gender, parental education, geographic remoteness, household composition, and Indigenous status.

*** Model 3: Association between 'tooth brushing habits' and 'Household income' or 'SEIFA score' (mutually adjusting for both 'household income' and 'SEIFA score' in the same model). Model also adjusted for: gender, parental education, geographic remoteness, household composition, and Indigenous status.

This article is protected by copyright. All rights reserved 


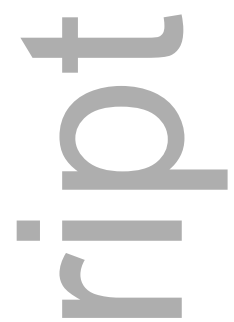

Table 4: Multivariable logistic regression models for the associations between household income, Socioeconomic Indexes for Areas (SEIFA) score and consumption of sugarsweetened beverages.

\begin{tabular}{|c|c|c|c|}
\hline \multirow[t]{2}{*}{ Observations ( $\mathrm{n}=2877)$} & Model $1 *$ & Model 2** & Model 3*** \\
\hline & OR $(95 \% \mathrm{CI})$ & OR $(95 \% \mathrm{CI})$ & OR $(95 \% \mathrm{CI})$ \\
\hline \multicolumn{4}{|l|}{ Household income } \\
\hline Quintile 1 (lowest income) & \multicolumn{2}{|l|}{1.00 (reference) } & 1.00 (reference) \\
\hline Quintile 2 & \multicolumn{2}{|l|}{$0.97(0.71,1.32)$} & $0.98(0.72,1.34)$ \\
\hline Quintile 3 & \multicolumn{2}{|l|}{$1.08(0.78,1.48)$} & $1.12(0.81,1.54)$ \\
\hline Quintile 4 & \multicolumn{2}{|l|}{$1.06(0.77,1.46)$} & $1.11(0.81,1.53)$ \\
\hline Quintile 5 (highest income) & \multicolumn{2}{|l|}{$0.65(0.48,0.87)$} & $0.71(0.53,0.97)$ \\
\hline \multicolumn{4}{|l|}{ SEIFA } \\
\hline \multicolumn{2}{|l|}{ Quintile 1 (most disadvantaged) } & 1.00 (reference) & 1.00 (reference) \\
\hline \multicolumn{2}{|l|}{ Quintile 2} & $0.88(0.65,1.21)$ & $0.90(0.65,1.23)$ \\
\hline \multicolumn{2}{|l|}{ Quintile 3} & $0.81(0.62,1.07)$ & $0.82(0.62,1.09)$ \\
\hline \multicolumn{2}{|l|}{ Quintile 4} & $0.81(0.60,1.09)$ & $0.85(0.62,1.15)$ \\
\hline \multicolumn{2}{|l|}{ Quintile 5 (least disadvantaged) } & $0.64(0.47,0.87)$ & $0.70(0.51,0.96)$ \\
\hline
\end{tabular}

This article is protected by copyright. All rights reserved 
*** Model 3: Association between 'consumption of sugar-sweetened beverages' and 'Household income' or 'SEIFA score' (mutually adjusting for both 'household income' and 'SEIFA score' in the same model). Model adjusted for: gender, parental education, geographic remoteness, household composition, and Indigenous status.

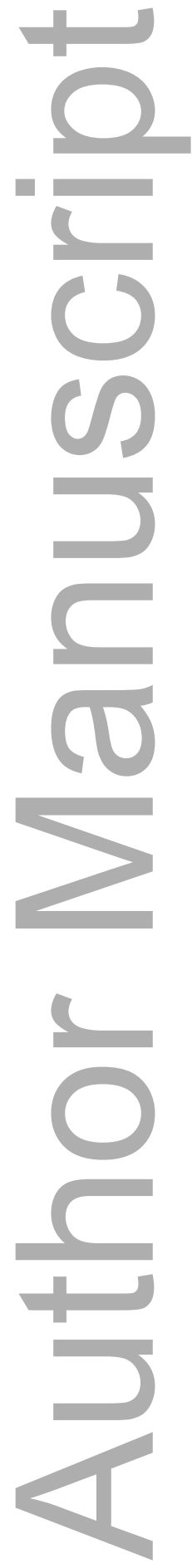




\section{University Library}

\section{- M M N E R VA A gateway to Melbourne's research publications}

Minerva Access is the Institutional Repository of The University of Melbourne

Author/s:

Kaunein, N;Singh, A;King, T

Title:

Associations between Individual-level and Area-level social disadvantage and oral health behaviours in Australian adolescents

Date:

2020-12

Citation:

Kaunein, N., Singh, A. \& King, T. (2020). Associations between Individual-level and Area-level social disadvantage and oral health behaviours in Australian adolescents.

AUSTRALIAN DENTAL JOURNAL, 65 (4), pp.286-293. https://doi.org/10.1111/adj.12792.

Persistent Link:

http://hdl.handle.net/11343/276350 